\title{
Physical Model Parameter Estimation of a Nonlinear Process
}

\author{
Limei Ding, Thomas Gustafsson and Andreas Johansson
}

\begin{abstract}
A physical model of a nonlinear subprocess in a continuous paper pulp digester is discussed and simplified. Model approximation is carried out in order to produce a simple linear model to be used for unknown parameter estimation of the physical model. The Taylor series expansion and the orthogonal collocation method are applied for the model linearization and model lumping, respectively. The reduced model is expressed as a standard state space form. The model parameters are estimated in the least squares sense, and the parameters retain their own physical meanings. The results of the parameter estimation are discussed and the model is verified using validation data.
\end{abstract}

\section{INTRODUCTION}

Process models play a more and more important role in the development of control technology. Modelling of a continuous paper pulp digester is a troublesome task due to a complex mixture of chemical reactions and transport phenomena within the process. System identification is a good alternative to model a complex process, for example, a digester. Funkquist [1], [2] used a grey-box identification approach for model parameter estimation of a continuous digester. Wisnewski et al. [3] applied an identification procedure based on normalized moments of an impulse response to identify a set of linear models used for the model predictive control of a continuous digester. Alexandridis [4] applied the partial least squares method in model identification based on a Purdue digester model proposed by Wisnewski et al. [5]. Amirthalingam et al. [6], [7] presented subspace identification based inferential control of a continuous pulp digester.

Comparing with black-box models, the parameters in a physical model bear certain physical meanings, which can be very useful in the diagnostic procedure or the controller design. The current study aims to deliver a simple linear model for a distributed parameter nonlinear process. For this process a complex nonlinear physical model is already available, and there exist unknown parameters in the physical model. The unknown parameters can be identified based on the simple linear model that is an approximation of the complex physical model, and the parameter estimates retain their own physical meanings. A subprocess in a continuous paper pulp digester is selected as an application example. In the next section, a physical digester model is simplified for the selected subprocess. Then the model is linearized and lumped. In Section 3, a software tool developed for the parameter estimation is applied and the parameter estimation results are analyzed. Model validation is discussed in Section 4. Section 5 gives conclusions.

Division of Systems and Interaction, Luleå University of Technology, SE-97187, Luleå, Sweden

\section{A NONLINEAR SUBPROCESS IN A DIGESTER}

A continuous paper pulp digester at Husum pulp mill, Mreal, Sweden is a dual vessel arranged digester. The main digester vessel can be divided into a co-current zone and a counter-current zone, as that shown in Fig. 1. Each zone can be modelled by coupled nonlinear partial differential equations (PDEs), that are constructed based on balance equations. Due to the model similarity between the two zones, we select only a single zone, the co-current zone, for the study of model approximation and unknown parameter estimation. The selected subprocess is denoted as $G$ in Fig. 1 .

\section{A. Process modelling}

In the last few decades, physical model studies of continuous paper pulp digesters have been frequently reported [5], [8], [9], [10], [11], [12]. Process modelling of the current study is based on a fundamental model proposed by Bhartiya [12], 2003. For this model, each volume in a digester is assumed to contain three phases: solid phase, entrapped liquor phase and free liquor phase. The combined solid phase and entrapped liquor phase is referred to as chip phase. The solid phase is assumed to consist of five components. The entrapped liquor and the free liquor are aqueous solution with six components. For more detail of the model please refer to the original paper (Bhartiya [12], 2003).

Kappa number is an important quality variable of pulp, and it is often chosen as the main controlled variable of a controlled digester system. This variable is related to the residue of lignin in pulp, thus the lignin content in solid phase

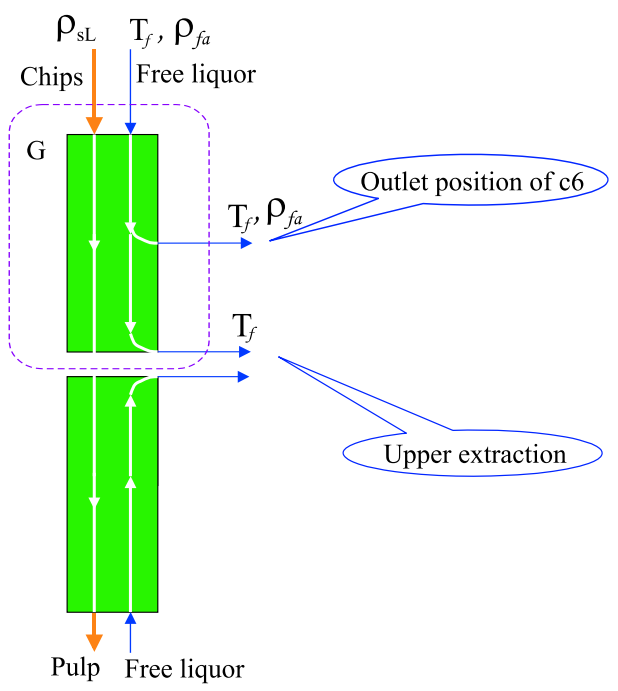

Fig. 1. A nonlinear sub-processes in a digester 
is an indirect measure of pulp quality along a continuous digester. The alkali concentration of entrapped liquor plays an important role in governing the rate of delignification. In addition, temperatures of substances involving delignification influence the reaction rate through the reaction rate constants. Therefore alkali concentration in liquor and temperatures of liquor and chips are often chosen as manipulated variables and observed variables.

From control and monitoring point of view, the following variables are important, and then they are selected as subprocess variables of $G$ :

$T_{f}$ temperature of free liquor phase $\left({ }^{\circ} C\right)$

$T_{c}$ temperature of chip phase $\left({ }^{\circ} \mathrm{C}\right)$

$\rho_{f a}$ concentration of alkali of free liquor phase $(\mathrm{g} / \mathrm{l})$

$\rho_{e a}$ concentration of alkali of entrapped liquor phase $(g / l)$

$\rho_{s L}$ concentration of lignin of solid phase ( $\%$ mass $O D W$ )

Based on the variable selection, the model proposed by Bhartiya is simplified. It differs from Bhartiya's model in that the lignin contained in chip, that includes high reactive lignin and low reactive lignin, is considered as a single component.

We make the following assumptions in order to obtain a simple model which describes some dominant dynamics of the subprocess.

\section{Assumptions:}

A1: The dynamic variations of chip porosity, the mass variations of solid and liquor, and the change in external volume flow rate can be neglected.

A2: The heat released by the exothermic reactions and energy transfer due to diffusion of components between the entrapped liquor and free liquor can be ignored.

A3: The process dynamics can be considered approximately linear around an equilibrium point.

A4: In the current study, only lignin in solid phase and effective alkali in liquor phase are taken into account in the reaction rate equations.

The simplified model is given as the following:

$$
\begin{aligned}
\frac{\partial T_{f}}{\partial t} & =-v_{f} \frac{\partial T_{f}}{\partial z}+d_{f}\left(T_{c}-T_{f}\right) \\
\frac{\partial T_{c}}{\partial t} & =-v_{c} \frac{\partial T_{c}}{\partial z}+d_{c}\left(T_{f}-T_{c}\right) \\
\frac{\partial \rho_{f a}}{\partial t} & =-v_{f} \frac{\partial \rho_{f a}}{\partial z}-D_{f}\left(\rho_{f a}-\rho_{e a}\right) \\
\frac{\partial \rho_{e a}}{\partial t} & =-v_{c} \frac{\partial \rho_{e a}}{\partial z}+D_{c}\left(\rho_{f a}-\rho_{e a}\right)+R_{e a} \\
\frac{\partial \rho_{s L}}{\partial t} & =-v_{c} \frac{\partial \rho_{s L}}{\partial z}+R_{s L}
\end{aligned}
$$

Comparing with the model in [12], here $D_{c}=D, D_{f}=$ $D \epsilon \frac{(1-\eta)}{\eta}, d_{c}=U, d_{f}=U \frac{1-\eta}{\eta}, k=e_{f} k_{1 L}$, and $\gamma$ is a stoichiometric coefficient. In the model $R_{s L}$ is the reaction rate of lignin of solid phase, and $R_{e a}$ is the reaction rate of alkali of entrapped liquor phase. The nonlinear relation of the reaction rates are given as follows:

$$
\begin{aligned}
R_{s L} & =-k \rho_{e a} \rho_{s L} \\
R_{e a} & =\gamma R_{s L}
\end{aligned}
$$

This simplified model (1) - (7) is the model derived by Lundquist [13].

\section{B. Model linearization}

A nonlinear relation in a process model can be linearized at an equilibrium point to describe the process local dynamics. We place a notation $\Delta$ before the notations of the process variables to define the local dynamics, for example, using $\Delta T_{f}$ for the variable $T_{f}$.

The nonlinear relations given in (6) and (7) are linearized by applying a Taylor series expansion. Substituting the linearized $R_{s L}$ and $R_{e a}$ into (5) and (4), respectively, a linearized model of $G$ is then obtained as:

$$
\begin{aligned}
\frac{\partial \Delta T_{f}}{\partial t}= & -v_{f} \frac{\partial \Delta T_{f}}{\partial z}+d_{f}\left(\Delta T_{c}-\Delta T_{f}\right) \\
\frac{\partial \Delta T_{c}}{\partial t}= & -v_{c} \frac{\partial \Delta T_{c}}{\partial z}+d_{c}\left(\Delta T_{f}-\Delta T_{c}\right) \\
\frac{\partial \Delta \rho_{f a}}{\partial t}= & -v_{f} \frac{\partial \Delta \rho_{f a}}{\partial z}+D_{f}\left(\Delta \rho_{e a}-\Delta \rho_{f a}\right) \\
\frac{\partial \Delta \rho_{e a}}{\partial t}= & -v_{c} \frac{\partial \Delta \rho_{e a}}{\partial z}+D_{c}\left(\Delta \rho_{f a}-\Delta \rho_{e a}\right) \\
& +\gamma k\left(a_{1} \Delta \rho_{e a}+a_{2} \Delta \rho_{s L}\right) \\
\frac{\partial \Delta \rho_{s L}}{\partial t}= & -v_{c} \frac{\partial \Delta \rho_{s L}}{\partial z}+k\left(a_{1} \Delta \rho_{e a}+a_{2} \Delta \rho_{s L}\right)
\end{aligned}
$$

where $a_{1}=\bar{\rho}_{s L}$ and $a_{2}=\bar{\rho}_{e a}$ since $\frac{\partial\left(\rho_{e a} \rho_{s L}\right)}{\partial \rho_{e a}}=\rho_{s L}$ and $\frac{\partial\left(\rho_{e a} \rho_{s L}\right)}{\partial \rho_{s L}}=\rho_{e a}$. Notations $\bar{\rho}_{s L}$ and $\bar{\rho}_{e a}$ are the equilibrium points of $\rho_{s L}$ and $\rho_{e a}$.

\section{A lumped linear model}

The orthogonal collocation (OC) method is one of the methods of Weighted Residuals [14]. It is commonly used to obtain a lumped model of a distributed parameter system for process identification, simulation and control [1], [2], [15], [16]. Advantages of applying the OC method for model reduction are explained in the references, e.g. in [15], [16].

The two temperature variables in (8) and (9) and three concentration variables in (10) - (12) are defined as state variables of the process:

$$
\begin{aligned}
x & =\left[\begin{array}{lllll}
x_{1} & x_{2} & x_{3} & x_{4} & x_{5}
\end{array}\right]^{T} \\
& =\left[\begin{array}{lllll}
\Delta T_{f} & \Delta T_{c} & \Delta \rho_{f a} & \Delta \rho_{e a} & \Delta \rho_{s L}
\end{array}\right]^{T}
\end{aligned}
$$

where the state vector $x$ is a function of the spatial variable $z$ and the time variable $t$.

Note that the spatial variable $z$ does not refer to the real physical length. It is normalized via a linear variable transformation and we define $z \in[0,1]$.

The following notation is introduced:

$$
\frac{\partial x(z, t)}{\partial t}=f\left(x(z, t), \frac{\partial x(z, t)}{\partial z}\right) \quad z \in[0,1]
$$


where $f$ is the linear relation defined by (8) - (12), and the boundary condition is given as:

$$
x(0, t)=u(t)
$$

where $u(t)$ is a vector of process inputs.

The input $u$ and the output $y$ of $G$ are defined as:

$$
\begin{aligned}
u & =\left[\begin{array}{lll}
u_{1} & u_{2} & u_{3}
\end{array}\right]^{T} \\
& =\left[\begin{array}{lll}
\Delta T_{s}\left(z_{t o p}, t\right) & \Delta \rho_{f a}\left(z_{t o p}, t\right) & \Delta \rho_{s L}\left(z_{t o p}, t\right)
\end{array}\right]^{T} \\
y & =\left[\begin{array}{lll}
y_{1} & y_{2} & y_{3}
\end{array}\right]^{T} \\
& =\left[\begin{array}{lll}
\Delta T_{f}\left(z_{c 6}, t\right) & \Delta \rho_{f a}\left(z_{c 6}, t\right) & \Delta T_{f}\left(z_{e x t}, t\right)
\end{array}\right]^{T}
\end{aligned}
$$

where $z_{t o p}, z_{c 6}$ and $z_{\text {ext }}$ denote the vertical positions of the digester at the top, the outlet of c6 and the upper extraction, respectively. Notation $T_{s}\left(z_{t o p}, t\right)$ denotes the top steam temperature. The selection of in- and output variables depends not only on the variable importance for the process observation and control, but also on the validity of the variable online measurement. For example, although the alkali concentration of the free liquor at extraction is important, it is not selected as a process output since its online measurement is not available.

A trial solution of $x(z, t)$ is chosen as the polynomial:

$$
x_{T}(z, t)=\sum_{j=0}^{N+1} x\left(z_{j}, t\right) l_{j}(z)
$$

where the basis functions $l_{j}(z)$ are Lagrange interpolation polynomials with the interpolation points chosen as the interior collocation points $z_{1}, z_{2}, \cdots, z_{N}$, and the boundary points $z_{0}=0$ and $z_{N+1}=1$.

The first order spatial derivative can then be approximated as:

$$
\frac{\partial x(z, t)}{\partial z} \approx \frac{\partial x_{T}(z, t)}{\partial z}=\sum_{j=0}^{N+1} \frac{\partial l_{j}(z)}{\partial z} x\left(z_{j}, t\right)
$$

For an arbitrary single state variable $x_{i}(z, t)$ in (13), where $i=1, \cdots, 5$, its spatial derivatives at the collocation points can be derived as the following:

$$
\begin{aligned}
& {\left[\begin{array}{l}
\left.\frac{\partial x_{i T}}{\partial z}\right|_{z_{1}} \\
\left.\frac{\partial x_{i T}}{\partial z}\right|_{z_{2}} \\
\ldots \\
\left.\frac{\partial x_{i T}}{\partial z}\right|_{z_{N+1}}
\end{array}\right]=\left[\begin{array}{c}
\left.\frac{\partial l_{0}}{\partial z}\right|_{z_{1}} \\
\left.\frac{\partial l_{0}}{\partial z}\right|_{z_{2}} \\
\ldots \\
\left.\frac{\partial l_{0}}{\partial z}\right|_{z_{N+1}}
\end{array}\right] x_{i}\left(z_{0}, t\right) } \\
+ & {\left[\begin{array}{ccc}
\left.\frac{\partial l_{1}}{\partial z}\right|_{z_{1}} & \ldots & \left.\frac{\partial l_{N+1}}{\partial z}\right|_{z_{1}} \\
\left.\frac{\partial l_{1}}{\partial z}\right|_{z_{2}} & \ldots & \left.\frac{\partial l_{N+1}}{\partial z}\right|_{z_{2}} \\
\vdots & \ldots & \vdots \\
\left.\frac{\partial l_{1}}{\partial z}\right|_{z_{N+1}} & \ldots & \left.\frac{\partial l_{N+1}}{\partial z}\right|_{z_{N+1}}
\end{array}\right]\left[\begin{array}{l}
x_{i}\left(z_{1}, t\right) \\
\vdots \\
x_{i}\left(z_{N+1}, t\right)
\end{array}\right] } \\
= & B_{1} x_{i}\left(z_{0}, t\right)+A_{1} x_{i}(t)
\end{aligned}
$$

The linearized model (8) - (12) can then be approximated by the OC approximation in a standard state space form:

$$
\begin{aligned}
& \dot{x}(t)=A x(t)+B u(t) \\
& y(t)=C x(t)+D u(t)
\end{aligned}
$$

where the matrices of the state space model are given as (22). The matrices $A_{1}$ and $B_{1}$ are given from (20). According to (18), matrices $C_{1}, C_{2}, D_{1}$ and $D_{2}$ are defined as the following:

$$
\begin{aligned}
C_{1} & =\left[\begin{array}{llll}
l_{1}\left(z_{c 6}\right) & l_{2}\left(z_{c 6}\right) & \cdots & l_{N+1}\left(z_{c 6}\right)
\end{array}\right] \\
C_{2} & =\left[\begin{array}{llll}
l_{1}\left(z_{N+1}\right) & l_{2}\left(z_{N+1}\right) & \cdots & l_{N+1}\left(z_{N+1}\right)
\end{array}\right] \\
D_{1} & =l_{0}\left(z_{c 6}\right) \\
D_{2} & =l_{0}\left(z_{N+1}\right)
\end{aligned}
$$

The identity matrix and zero matrix in $A$ has the same dimension as $A_{1}$, and the zero matrices in matrices $B, C$ and $D$ have the same dimensions as matrices $B_{1}, C_{1}$ and $D_{1}$, respectively.

\section{UNKNOWN PARAMETER ESTIMATION OF THE PHYSICAL MODEL}

A vector of unknown parameters exist in (8)-(12) is defined as:

$$
\theta=\left[\begin{array}{llllllllll}
v_{f} & d_{f} & v_{c} & d_{c} & D_{f} & D_{c} & k & \gamma & a_{1} & a_{2}
\end{array}\right]
$$

In the least-squares sense, the model agreement resulted from the parameter estimation is indicated using a loss function $V(\theta)$ defined as:

$$
V(\theta)=\sum_{j=1}^{3} \sum_{i=1}^{n}\left(y_{j s}(i)-y_{j m}(i)\right)^{2}
$$

where $y_{j s}$ is the $j t h$ simulated output, $y_{j m}$ is the $j t h$ measured output and $n$ is the number of data points.

A software tool developed for the model parameter estimation is applied. The software package consists of program routines encoded in MATLAB 7.0.1, which are divided into three categories to accomplish the following tasks:

1) Model linearization, finite dimensional approximation, and model implementation.

2) Process variable selection, data processing, process simulation.

3) Loss function definition, parameter start point selection, parameter estimation and model validation.

\section{A. The influence of $u_{3}$ on the identification process}

The data of most input and output signals selected in (16) and (17) are collected from their on-line measurements. However, the signal $u_{3}=\Delta \rho_{s L}\left(z_{t o p}, t\right)$ is not measured online, that causes a lack of input data collection. According to the experience and the knowledge of the process, the variation of lignin content at the top of digester is very slow, therefore the input signal $u_{3}$ is assumed as a constant. Unknown parameter estimation started from a guess of parameter start point. In order to test the influence from the variation of $u_{3}$, we keep the parameter start point unchanged, then increase the value of $u_{3}$.

It can be seen from (11) and (12) that the sensitivity of the model response to the change of $u_{3}$ depends on the parameter values of $\gamma$ and $a_{2}$. We use two guessed start points of the parameters in the test. The first one is denoted as $\theta_{S P 1}$, in 


$$
\begin{aligned}
& A=\left[\begin{array}{ccccc}
-v_{f} A_{1}-d_{f} I & d_{f} I & 0 & 0 & 0 \\
d_{c} I & -v_{c} A_{1}-d_{c} I & 0 & 0 & 0 \\
0 & 0 & -v_{f} A_{1}-D_{f} I & D_{f} I & 0 \\
0 & 0 & D_{c} I & -v_{c} A_{1}-\left(D_{e}-\gamma k a_{1}\right) I & \gamma k a_{2} I \\
0 & 0 & 0 & k a_{1} I & -v_{c} A_{1}+k a_{2} I
\end{array}\right] \\
& B=\left[\begin{array}{ccc}
-v_{f} B_{1} & 0 & 0 \\
-v_{c} B_{1} & 0 & 0 \\
0 & -v_{f} B_{1} & 0 \\
0 & -v_{c} B_{1} & 0 \\
0 & 0 & -v_{c} B_{1}
\end{array}\right] \\
& C=\left[\begin{array}{ccccc}
C_{1} & 0 & 0 & 0 & 0 \\
0 & 0 & C_{1} & 0 & 0 \\
C_{2} & 0 & 0 & 0 & 0
\end{array}\right] \\
& D=\left[\begin{array}{ccc}
D_{1} & 0 & 0 \\
0 & D_{1} & 0 \\
D_{2} & 0 & 0
\end{array}\right]
\end{aligned}
$$

TABLE I

PARAMETER ESTIMATIONS WITH DIFFERENT VALUES OF CONSTANT INPUT $u_{3}=\Delta \rho_{s L}($ top $)$.

\begin{tabular}{rccccccc}
\hline & $u_{3}:$ & 1 & 2 & 3 & 5 & 10 & 20 \\
\hline$\theta_{S P 1}$ & No. & 1 & 2 & 3 & 4 & 5 & 6 \\
Start $V(\theta):$ & 1618.86 & 1618.86 & 1618.86 & 1618.86 & 1618.86 & 1618.86 \\
Final $V(\theta):$ & 965.632 & 965.684 & 967.021 & 965.761 & 965.7 & 965.641 \\
No. I/FC: & $27 / 308$ & $33 / 374$ & $35 / 396$ & $33 / 374$ & $63 / 704$ & $22 / 253$ \\
\hline$\theta_{S P 2}$ & No. & 1 & 2 & 3 & 4 & 5 & 6 \\
Start $V(\theta):$ & 7052.38 & 122350 & 47211.4 & 1125624 & 489014 & 1933050 \\
Final $V(\theta):$ & 966.124 & 966.252 & 965.6911 & 965.784 & 992.586 & 999.877 \\
No. I/FC: & $18 / 209$ & $45 / 506$ & $68 / 759$ & $210 / 2321$ & $465 / 5126$ & $34 / 385$ \\
\hline
\end{tabular}

which the values of $\gamma$ and $a_{2}$ are relative small. Increasing the two parameter values, the second start point is denoted as $\theta_{S P 2}$. The identification process must be more sensitive to the variation of $u_{3}$ using $\theta_{S P 2}$ than using $\theta_{S P 1}$. Table I shows some data dealing with the identification process: different choice of $u_{3}$, start value and final value of the loss function $V(\theta)$, and the number of iteration/function count (No. I/FC).

Observing the identification process with $\theta_{S P 1}$, the start value of $V(\theta)$ is not influenced by the variation of $u_{3}$ because of small start values of $\gamma$ and $a_{2}$. With $\theta_{S P 2}$, the increase of $u_{3}$ strongly influences the start value of $V(\theta)$, and it makes the parameter estimation difficult by ether requiring a big iteration number or resulting a great final $V(\theta)$. This implies that it takes longer time to estimate parameter for a larger $u_{3}$ or the parameter search ends at a bad local optimal value. A negative big value of $u_{3}$ also leads problems, and it is not discussed here in detail due to the page limit.

In fact a large $u_{3}$ is unreasonable since a local linearized model only allow small signal variations, and in general the top lignin content in chip $\left(\rho_{s L}\left(z_{t o p}\right)\right)$ does not vary so much. In summary, selecting a small value of lignin content change at the top of the digester, e.g., $\Delta \rho_{s L}\left(z_{t o p}\right) \in[1,3]$, is a reasonable choice for $u_{3}$.

\section{B. Identification with varied start points}

Choosing $u_{3}=1$ and keeping this input fixed, the parameter estimation with different start points of the parameters is performed. We repeat the estimation, and each time a new start point is applied. Looking at the final parameter estimates obtained with 7 different start points, the estimation results do not depend on varied choice of start point. The most estimations are ended with the final $V(\theta)<966$ and only one estimation has final $V(\theta)=968.188$.

We select the estimations with final $V(\theta)>966$, i.e., one estimation mentioned in the former paragraph, and the estimations No.5 and No.6 with $\theta_{S P 2}$ shown in Table I, then re-identify the parameters after a fine adjustment of start points. Improved results of the selected estimations are obtained in which the final values of $V(\theta)$ in the reidentifications approach the normal level. This implies that an estimation could go in a wrong direction such that the parameter search is ended in a local optimum. A fine tuning of start point may affect the search direction so that a better optimal estimate can be achieved. However, the numbers of $\mathrm{I} / \mathrm{FC}$ in the re-identification for No.5 and No.6 with $\theta_{S P 2}$ in Table I are 105/1166 and 1427/15708 respectively, and this still supports the discussion made in subsection III-A: a big value of $u_{3}$ makes parameter estimation difficult.

\section{Estimation results analysis}

A larger value of final $V(\theta)$ implies a greater residue in an estimation. In contrast, an estimation with a less final $V(\theta)$ often provides a better estimate. Collecting the estimates with the final $V(\theta)<966$ from the identification results given in subsection III-A and subsection III-B, then plot the estimates of parameters $v_{f}, d_{f}, v_{c}, d_{c}, D_{f}, D_{c}, a_{1}$, and $a_{2}$ in Fig. 2, where the mean value of the estimates for each parameter is 
illustrated using a solid line. The dotted lines illustrate the $\pm 5 \%$ interval around the mean value, and the dashed lines are $\pm 10 \%$ interval. The $\pm 20 \%$ interval corresponds to the vertical axes.

All the estimates of velocities $v_{f}$ and $v_{c}$ converge to each own single parameter value and the deviations of the two estimates from their mean values are zero. Parameters $d_{f}$ and $d_{c}$ are coefficients that concern the interphase heat transfer, and relate only the linear part of the simplified model. The deviations of the estimates of $d_{f}$ and $d_{c}$ from their mean values do not exceed $3 \%$ interval.

However, the process nonlinear properties strongly affects the dynamics of $\rho_{e a}$ and $\rho_{s L}$, then further affects $\rho_{f a}$ due to the connection between (10) and (11). Thus the estimation of the rest parameters may be deteriorated, since the parameter estimation is based on an approximate linear representation of the nonlinear relation. Parameters $D_{f}$ and $D_{c}$ are coefficients concerning the mass transfer between $\rho_{f a}$ and $\rho_{e a}$. In fact, the estimates of $D_{f}$ and $D_{c}$ distribute less closely around their mean values. There are $64 \%$ estimates of $D_{f}$ and near $30 \%$ estimates of $D_{c}$ distribute within $10 \%$ interval and almost all estimates of $D_{f}$ and $D_{c}$ are placed within $20 \%$ interval.

Parameters $a_{1}$ and $a_{2}$ are defined due to the model linearization, and they are given as:

$$
a_{1}=\bar{\rho}_{s L} \quad a_{2}=\bar{\rho}_{e a}
$$

The mean value of $a_{1}$ in Fig.2 is 28.839. Comparing it with the real work point of lignin content at top which is 29 (\% mass $O D W)$, this estimate is reasonable. All the estimates of $a_{2}$ in Fig.2 give a zero value. Although signal $\rho_{e a}$ does not measured online, its value can not exceed the upper limit of $\rho_{f a}$. In fact the measured $\rho_{f a}$ is processed to match the dimension of $\rho_{s L}$, and $\rho_{f a}$ is much less than $\rho_{s L}$ when the two signals are compared with same dimension. This results $\bar{\rho}_{f a} \ll \bar{\rho}_{s L}$, then we have $\bar{\rho}_{e a} \ll \bar{\rho}_{s L}$. The estimates of $a_{1}$ and $a_{2}$ are acceptable since $a_{2} \ll a_{1}$.

Because of the product $k \cdot \gamma$ in (11), estimates of $k$ and $\gamma$ vary considerably. There is an inverse relationship concerning the value variations between $|k|$ and $\gamma$, and it is shown in the left part of Fig.3. A greater $|k|$ combined with a smaller $\gamma$ or a smaller $|k|$ combined with a greater $\gamma$ may give similar model accuracy.

Model agreement with measured data is demonstrated by the right plots in Fig. 3, where estimate of No.5 in Fig. 2 is applied.

\section{Model VALidation}

The mean squares error (MSE) of a process output is defined as:

$$
\operatorname{MSE}\left(y_{j}\right)=\frac{1}{n}\left(\sum_{i=1}^{n} \varepsilon_{j}^{2}(i)\right)^{\frac{1}{2}}
$$

where $\varepsilon_{j}$ denotes the residue of one of the process outputs:

$$
\varepsilon_{j}(i)=y_{j s}(i)-y_{j m}(i), \quad j=1,2,3 .
$$
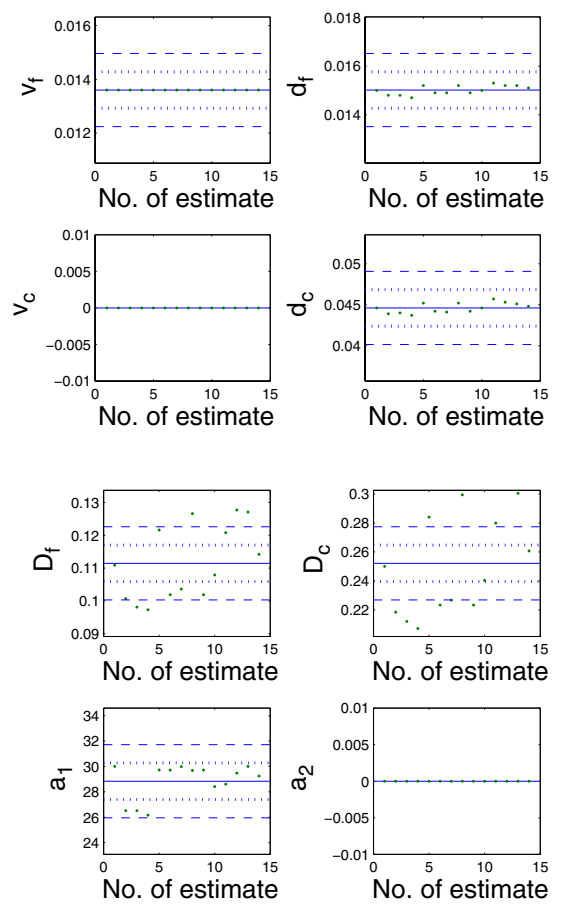

Fig. 2. Parameter estimates and their mean values
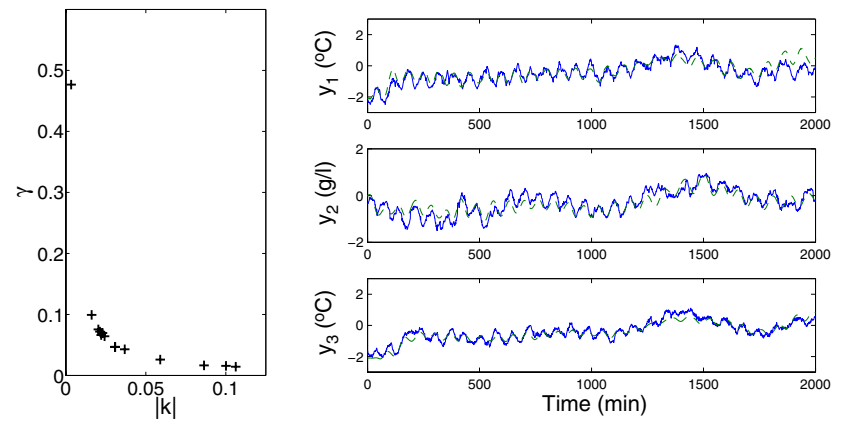

Fig. 3. Left: an inverse relationship concerning the value variations between $|k|$ and $\gamma$; right: measurements (solid line) and simulations (dashed line) using identification data.

and $n$ is the number of data points. The MSE of the identification results are shown in Table II. The values of MSE using both identification data and validation data are closed each other.

Fig. 4 demonstrates the simulation and the measured data of $G$ using validation data, where for the simulation the same parameter estimate as using for the simulation shown in Fig 3 is applied. Comparing with Fig. 3, there is no obvious deterioration of agreement between the measurement and the simulation using validation data.

From a point of view of control oriented model validation[17], a model error model can be identified based on the measured process data and the model residual data. Due to the page limit, we only illustrate a model error model in Fig. 5, that describe the model error concerning the output $y_{3}$. The model error model is a high-order black-box model 
TABLE II

MEAN SQUARES ERROR OF IDENTIFICATION RESULTS

\begin{tabular}{clrll}
\hline Data: & \multicolumn{2}{l}{ Identification } & \multicolumn{2}{c}{ Validation } \\
\hline \multirow{3}{*}{ MSE: } & $y_{1}$ & 0.0055 & $y_{1}$ & 0.0048 \\
& $y_{2}$ & 0.0040 & $y_{2}$ & 0.0044 \\
& $y_{3}$ & 0.0037 & $y_{3}$ & 0.0040 \\
\hline
\end{tabular}
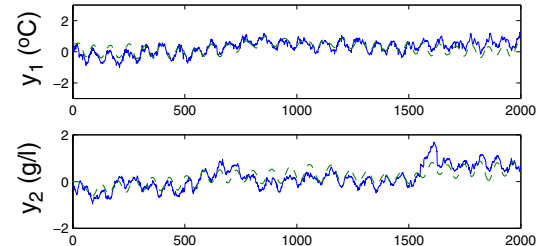

$$
\text { OO }
$$

Fig. 4. Measurements (solid line) and simulations (dashed line) using validation data.

identified using a black-box identification toolbox [18], and it provides helpful information for the controller design with robustness considerations.

\section{CONCLUSions}

A nonlinear subprocess of a digester is selected for the parameter estimation study. The model of the subprocess consists of nonlinear coupled PDEs. The nonlinear model is linearized and then reduced using the OC method in linear state space form. A software tool developed for model identification of the distributed parameter system is applied. The unknown parameter estimation of the selected process is based on a reduced physical model, thus the estimates of each parameter retain its own physical meaning.

The influences from the changes of a constant input and varied parameter start points on the identification process are tested and discussed. Some estimation results are improved by adjusting their parameter start points. The parameter estimates are illustrated graphically and discussed. The obtained model is verified by simulation using validation data and mean square error analysis, and also a model error model description. The identification provides acceptable parameter estimates for the reduced linear model that is suitable to describe the local dynamic properties of subprocess $G$.
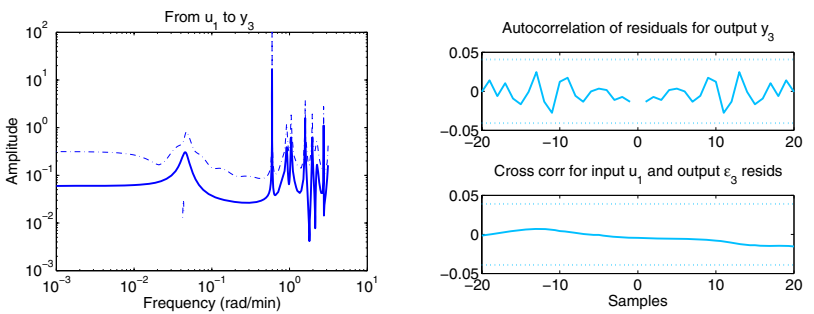

Fig. 5. Left: frequency response of a high order black-box model error model and its $99 \%$ confidence interval; right: correlation functions.
In the future the parameter identification will be carried out based on a more complex model.

\section{ACKNOWLEDGMENTS}

The authors would like to thank researchers at NPI, Mid Sweden University; at Eurocon; and at More Research, for their support and helpful discussions.

\section{REFERENCES}

[1] Funkquist, J. Modeling and identification of a distributed parameter process: the continuous digester. $\mathrm{PhD}$ thesis, Automatic Control, Department of Signals, Sensors and Systems, Royal Institute of Technology, Sweden, 1995.

[2] J. Funkquist, Grey-box identification of a continuous digester - a distributed-parameter process, Control Engineering Practice, vol.5, no.7, 1997, pp 919-930.

[3] P.A. Wisnewski, F.J. Doyle III, Control structure selection and model predictive control of the Weyerhaeuser digester problem, Journal of Process Control, vol.8, no.(5-6), 1998, pp 487-495.

[4] A. Alexandridis, H. Sarimveis, A. Angelou, T. Tetsina, G. Bafas, A model predictive control scheme for continuous pulp digesters based on the partial least square (PLS) modelling algorithm, In Proceedings of the 10th Control Systems conference, June 3-5, 2002, Stockholm, Sweden, pp $253-257$.

[5] P.A. Wisnewski, F.J. Doyle III, and F. Kayihan. Fundamental continuous pulp digester model for simulation and control, AIChE J., vol. 43, 1997, pp 3175-3192.

[6] R. Amirthalingam, J.H. Lee, Subspace identification based inferential control applied to a continuous pulp digester, Journal of process control, vol.9, 1999, pp 397-406.

[7] R. Amirthalingam, J.H. Lee. Subspace identification based inferential control of a continuous pulp digester, Computers \& Chemical Engineering, vol.21, 1997, pp 1143-1148.

[8] R.R. Gustafson, C.A. Sleicher, W.T. McKean, B.A. Finlayson, Theoretical model of a Kraft pulping process, Ind. Eng. Chem. Process Des. Dev., vol.22, 1983, pp 87-96.

[9] A.C. Butler, T.J. Williams. A Description and User's Guide for the Purdue Kamyr Digester Model. Technical Report 152, Purdue University, PLAIC, Purdue Engineering, West Lafayette, IN 47907, December, 1988.

[10] F.A. Michelsen. A dynamic mechanistic model and model-based analysis of continuous Kamyr digester, PhD Thesis, 1995 Report no. 95-4-W, University of Trondheim, 1995.

[11] F. Kayihan, M.A. Gelormino, E.M. Hanczyc, F.J. Doyle III, Y. Arkun. A Kamyr continuous digester model for identification and controller design, Proc. IFAC World Cong. San Francisco, Elsevier Acience Publishers, New York, 1996, pp M37-42.

[12] S. Bhartiya, P. Dufour, F.J. Doyle III, Fundamental thermal-hydraulic pulp digester model with grade transition, AIChE Journal, vol. 49, no. 2, 2003, pp 411-425.

[13] S.O. Lundqvist, Matematisk modell av kontinuerlig sulfatkokare. In SCAN FORSK, Vol. 72. Stockholm, Sweden: STFI. In Swedish. 1975.

[14] J. Villadsen, M. Michelsen, Solution of Differential Equation Models by Polynomial Approximation, Prentice-Hall, Englewood Cliffs, NJ, 1978.

[15] D. Dochain, J.P. Babary, N. Tali-Maanar, Modeling and adaptive control of nonlinear distributed parameter bioreactors via orthogonal collocation, Automatica, vol.28, no.5, 1992, pp 873-883.

[16] S. Bourrel, D. Dochain, J.P. Babary, I. Queinnec, Modelling, identification and control of a denitrifying biofilter, Jurnal of Process Control, Vol.10, 2000, pp 73-91.

[17] Ljung, L. (1999). Model validation and model error modeling. In B. Wittenmark, \& A. Rantzer (Eds.), Proceedings of the Åström symposium on control, Studentliteratur, Lund, Sweden (pp. 15-42).

[18] MathWorks, System Identification Toolbox. 